\title{
The GTPase-activating protein Rap1GAP: A new player to modulate Ret signaling
}

\author{
Gustavo Paratcha ${ }^{1,2}$, Fernanda Ledda ${ }^{1,2}$ \\ ${ }^{I}$ Division of Molecular and Cellular Neuroscience, Institute of Cellular Biology and Neuroscience Prof. Dr. E. De Robertis (IBCN)- \\ CONICET, School of Medicine, University of Buenos Aires, Buenos Aires, Argentina; ${ }^{2}$ Laboratory of Molecular and Cellular Neu- \\ roscience, Department of Neuroscience, Karolinska Institute, Stockholm, Sweden
}

Cell Research (2011) 21: 217-219. doi:10.1038/cr.2010.143; published online 19 October 2010

Glial cell line-derived neurotrophic factor (GDNF) plays a critical role in orchestrating the development and maintenance of different populations of central and peripheral neurons. GDNF was initially discovered by its ability to promote the survival of ventral midbrain dopaminergic neurons [1]. In addition, GDNF promotes the survival and control the differentiation of motor neurons and many peripheral neurons, including sensory and sympathetic neurons. Moreover, it is required to induce proliferation, migration and survival of enteric neurons. GDNF promotes these trophic effects through the activation of the receptor tyrosine kinase (RTK) Ret. A distinctive feature of the receptor complex for GDNF is the requirement of two types of receptors, one specialized in GDNF binding, represented by the glycosyl-phosphatidyl inositol (GPI)-linked co-receptor GFR $\alpha 1$, and another involved in transmembrane signaling, represented by the RTK Ret or the neural cell adhesion molecule NCAM [1, 2]. Following homodimeric GDNF binding to GFR $\alpha 1$, Ret becomes dimerized and tyrosine phosphorylated, and triggers many differ-

Correspondence: Fernanda Ledda E-mails: fernanda.ledda@ki.se;mledda@fmed. uba.ar ent signaling pathways, including the Ras-Raf-MAPK (ERK1/2) cascade, the phosphatidylinositol-3-kinase (PI3K)Akt, the PLC- $\gamma$ and the Src signaling pathways [3].

To gain a better understanding of Ret signaling, Jiao et al. [4] performed a yeast two-hybrid assay to screen for novel Ret interactors using the intracellular domain of Ret as bait. In this paper, the authors describe the identification of Rap1GAP, a GTPase-activating protein (GAP) for Rap1, as a novel Ret-binding protein. Interestingly, this result provides the first evidence for a direct interaction between Rap1GAP with a RTK. In this elegant study, the authors also demonstrate that endogenous Rap1GAP specifically associate with Ret in midbrain and spinal cord in vivo. By systematically comparing five different tyrosine phosphorylation sites (Tyr905, Tyr981, Tyr1015, Tyr1062 and Tyr1096) within the intracellular domain of Ret (Ret $\left.{ }^{\mathrm{ICD}}\right)$, Jiao et al. identify the Tyr981 as the crucial docking site for Ret-Rap1GAP association.

Rap1 is a Ras-like small GTPase, which is activated by different extracellular factors and has been involved in diverse processes, such as cell adhesion, cell-cell junction formation and neuronal differentiation [5]. Rap1, as others small GTPases cycles between an inactive GDP-bound state and an active GTP-bound state. While guanine nucleotide exchange factors (GEFs) stimulate the exchange of GDP to GTP to generate the activated form of Rap1, GTPases activating proteins, like Rap$1 \mathrm{GAP}$, increase the intrinsic rate of GTP hydrolysis inactivating Rap1. Thus the activation state of Rap1 depends on the balance between Rap1GEFs and Rap1GAPs.

Despite considerable advances in understanding the signaling pathways through which Ret controls neuronal survival and proliferation, the molecular determinants underlying Ret-mediated neuronal differentiation are still much less known. It has been established that ERK $1 / 2$ activation by growth factors can induce cell growth and/or differentiation. Although transient activation of ERK1/2 is thought to promote proliferation, its sustained activation appears to induce morphological differentiation. In the PC12 cell line, the neurotrophin nerve growth factor (NGF) promotes a robust neurite outgrowth through a sustained ERK1/2 activation mediated by the activation of Rap1 [6]. Taking advantages of this cellular model, Jiao et al. show that Rap1GAP inhibits GDNF/Ret-induced neurite outgrowth by restricting both the activation of Rap1 (Rap1-GTP) and the sustained activation of ERK1/2 [4]. These findings are in agreement with previous data 


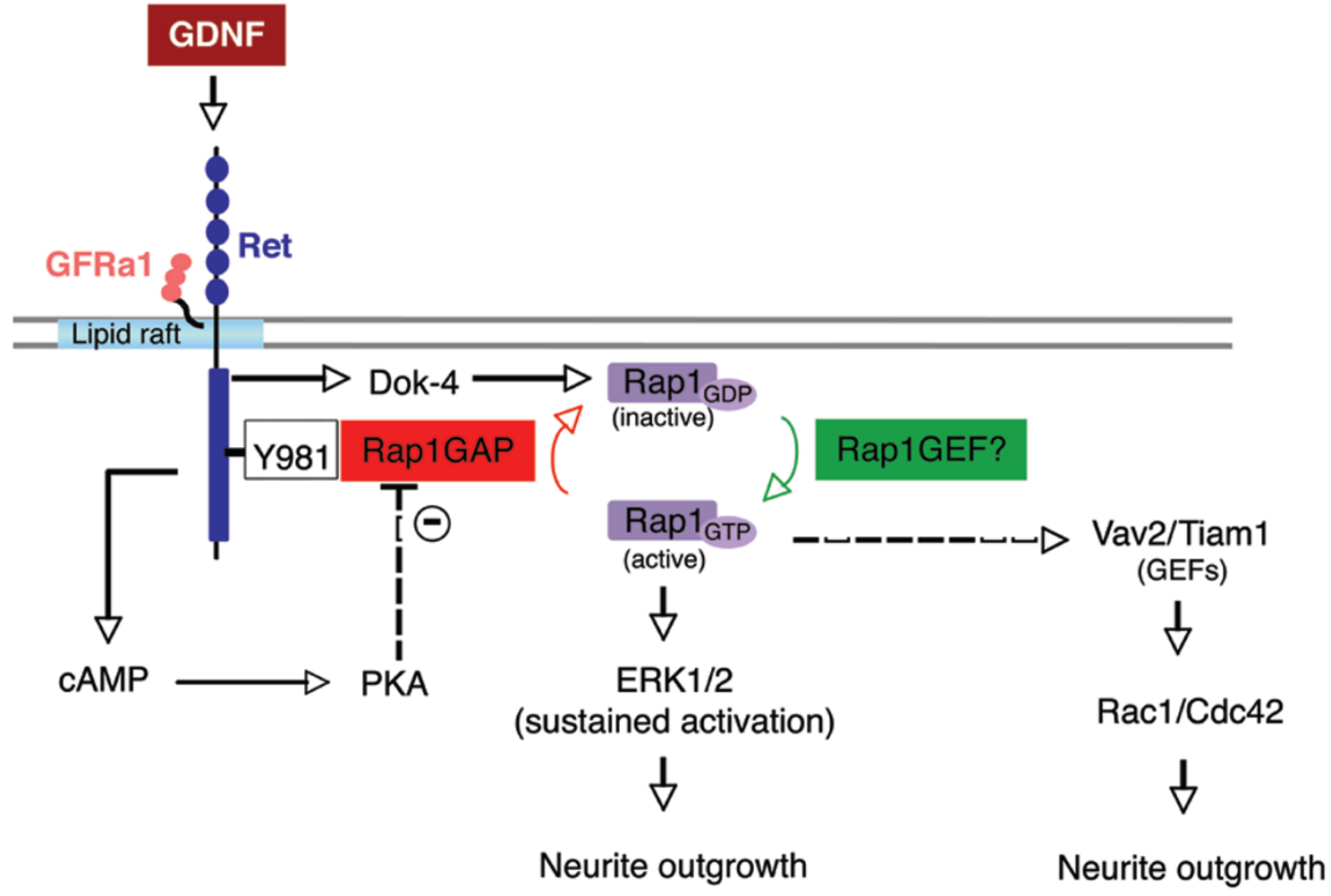

Figure 1 Model describing the proposed role of Rap1GAP in the control of GDNF-induced Ret signaling. After activation of Ret by the heterocomplex GDNF/GFRa1, Ret becomes phosphorylated at different tyrosine sites. In particular, the Tyr1062 has been described to mediate the activation of Dok-4, which then triggers a sustained activation of Rap1-ERK1/2 pathway and neurite outgrowth. At the same time, Ret activation results in phosphorylation of the Tyr981 (Y981), which is the docking site involved in the recruitment of the negative signaling regulator Rap1GAP. Thus, Rap1GAP counterbalances the Dok-4/Rap1/ ERK1/2 pathway and controls neurite outgrowth. The figure also shows a possible role of PKA in the inhibition of Rap1GAP activity (dashed line), and indicates the potential involvement of Rap1 in the modulation of neurite outgrowth through the concomitant activation of Vav2 and/or Tiam1, two GEFs for Rac1 and Cdc-42 (dashed arrow).

showing that GDNF-promoted neurite outgrowth during neuronal development involves prolonged activation of Rap1-ERK1/2 pathway via the adaptor protein, Dok-4, which is phosphorylated after Ret activation [7]. Interestingly, Dok-4 was identified as a direct interactor partner of Ret by yeast two-hybrid screen [8]. In this work, the authors show that Dok-4 can directly associate with Tyr1062. Together, these results indicate that after ligand binding, Ret becomes phosphorylated in different sites including Tyr1062 and Tyr981. While phosphorylation of Tyr1062 mediates the activation of Dok-4, which then triggers a sustained activation of Rap1-ERK1/2 pathway and neurite outgrowth, phosphorylation of Tyr981 mediates the recruitment of Rap1GAP, a negative regulator of Rap1 signaling (see Figure 1).

A recent study identified Rap1GAP as a prominent PKA substrate. In this study, the authors show that the phosphorylation of Rap1GAP by PKA is associated with the inhibition of Rap1GAP activity [9]. As it is known, activation of Ret by GDNF elevates cytoplasmic levels of cAMP and activates PKA in neuronal cells [10]. These observations suggest that Ret induces a sustained Rap1-ERK1/2 pathway probably by a dual mechanism that involves (i) Dok-4 mediated Rap1GEF activation and (ii) inhibition of Rap1GAP activity by sequestering and blocking its GAP activity through a PKA-dependent phosphorylation (Figure 1).

While the study by Jiao et al. provides new insights into the molecular mechanisms through which Rap1GAP limits Ret-induced neurite outgrowth, some questions still remain unanswered. Thus, how the Rap1GAP activity is modulated upon binding to Ret and how Rap1GAP competes with other adaptor proteins, like Src, which has been described to bind Tyr981 [11], are important questions that require additional analysis. Interestingly, Rap1 plays an important role in the regulation of the cytoskeleton by activating the Rho GTPase family members Rac1/Cdc42 via the binding and recruitment of the GEFs Tiam1 and Vav2 [12]. Therefore, another issue that would need to be 
addressed is whether Rap1GAP may also collaborate in the control of additional downstream signaling pathways involved in Ret-dependent neurite outgrowth, such as activation of Rho GTPases.

The pleiotropic roles played by Ret during development and the complex intracellular network of signaling pathways triggered by Ret, suggested that Ret as other RTK, requires the existence of cellular mechanisms that control its activity, avoiding thereby abnormal cellular behavior and disease. Indeed, the picture that arise from the last years indicates that this receptor is tightly regulated in a cell-specific manner through the coordinated action of different protein inhibitors that function at multiple levels of the signaling cascade and at different time points after receptor engagement. Examples of Ret negative modulators include the ERK1/2 antagonist, sprouty [13] and the leucine-rich repeat and Ig-like protein, Lrig1 [14].

The physiological requirement of Ret signaling for the survival, maintenance and regeneration of the dopaminergic system supports additional investigation toward optimizing the ongoing GDNF clinical trials for Parkinson disease (PD) using activators or reducing the effects of endogenous antagonists of Ret signaling. Unlike Ret positive signaling effectors, which are relatively well understood, signaling attenuation is under intensive study. In this regard, the study performed by Jiao et al. adds a new physiological mechanism to negatively regulate signaling and biological responses induced by GDNF and Ret in neuronal cells. In particular, the fact that Ret is coexpressed with Rap1GAP in midbrain dopaminergic neurons together with the association between both molecules in midbrain tissue argue that this mechanism could have a potential therapeutic value for treatment of PD.

\section{References}

1 Lin LF, Doherty DH, Lile JD, Bektesh S, Collins F. GDNF: a glial cell linederived neurtrophic factor for midbrain dopaminergic neurons. Science 1993; 260:1130-1132.

2 Paratcha G, Ledda F. GDNF and GFRalpha: a versatile molecular complex for developing neurons. Trends Neurosci 2008; 8:384-391.

3 Airaksinen MS, Saarma M. The GDNF family: signaling, biological functions and therapeutic value. Nat Rev Neurosci 2002; 5:383-394.

4 Jiao L, Zhang Y, Hu C, Wang YG, Huang A, He C. Rap1GAP interacts with Ret and suppresses GDNF-induced neurite outgrowth. Cell Res 2011; 21:327-337.

5 Kooistra M, Dubé N, Bos JL. Rap1: a key regulator in cell-cell junction formation. J Cell Sci 2007; 120(Pt 1):1722.

6 York RD, Yao H, Dillon T, et al. Rap1 mediates sustained MAPK activation induced by nerve growth factor. Nature 1998; 392:622-626.

7 Uchida M, Enomoto A, Fukuda T, et al. Dok-4 regulates GDNF-dependent neurite outgrowth through downstream activation of Rap1 and mitogen-activated protein kinase. J Cell Sci 2006; 119:3067-3077.

8 Grimm J, Sachs M, Britsch S, et al. Novel p62 family members, dok4 and dok5, are substrates of the c-Ret receptor tyrosine kinase and mediate neuronal differentiation. J Cell Biol 2001; 154:345-354

9 McAvoy T, Zhou M, Greengard P, Nairn AC. Phosphorylation of Rap1GAP, a striatally enriched protein, by protein kinase A controls Rap1 activity and dendritic spine morphology. Proc Natl Acad Sci USA 2009; 106:35313536.

10 Cai D, Shen Y, De Bellard M, Tang S, Filbin MT. Prior exposure to neurotrophins blocks inhibition of axonal regeneration by MAG and myeling via a cAMP-dependent mechanism. Neuron 1999; 2:89-101.

11 Encinas M, Ceowder RJ, Milbrandt J, Johnson EM Jr. Tyrosine 981, a novel ret autophosphorylation site, binds cSrc to mediate neuronal survival. J Biol Chem 2004; 279:18262-18269.

12 Arthur WT, Kuilliam LA, Kooper JA. Rap1 promotes cell spreading by localizing Rac1 nucleotide exchange factor. J Cell Biol 2004; 167:111-122.

13 Ishida M, Ichihara M, Mii S, et al. Sprouty2 regulates growth and differentiation of human neuroblastome cells through Ret tyrosine kinase. Cancer Sci 2007; 98:815-821.

14 Ledda F, Bieraugel O, Fard SS, Vilar M, Paratcha G. Lrig1 is an endogenous inhibitor of Ret receptor tyrosine kinase activation, downstream signaling, and biological responses to GDNF. $J$ Neurosci 2008; 28:39-49. 